\title{
MUTUAL POTENTIAL OF HOMOGENEOUS POLYHEDRA
}

\author{
ROBERT A. WERNER ${ }^{1}$ AND DANIEL J. SCHEERES ${ }^{2}$ \\ ${ }^{1}$ Jet Propulsion Laboratory, California Institute of Technology, 4800 Oak Grove Drive, \\ Pasadena, CA 91109-8001, USA, e-mail: robert.a.werner@jpl.nasa.gov \\ ${ }^{2}$ Department of Aerospace Engineering, The University of Michigan, 1320 Beal Avenue, Ann \\ Arbor, MI 48109-2140,USA, e-mail: scheeres@umich.edu
}

(Received: 2 May 2004; revised: 8 September 2004; accepted: 8 October 2004)

\begin{abstract}
The mutual gravitational potential between a pair of homogeneous polyhedra is expressed using an infinite series. The nested volume integrals are evaluated analytically and result in simple tensor expressions containing no special functions. However, complexity increases as $O\left(6^{n}\right)$, where $n$ is the term degree. An alternate formulation due to Liebenthal is also presented.
\end{abstract}

Key words: inertia integral, mutual potential, polyhedron potential, two rigid bodies problem

\section{Introduction}

We have been investigating the determination of the mutual gravitational potential. Among other applications, such expressions are necessary for investigating the coupled orbital and rotational dynamics of a binary asteroid system.

To date, there have been no general, closed-form solutions for the mutual potential. A series expansion formalism seems necessary.

For celestial bodies, the mutual potential has been expressed using spherical harmonics. Borderies (1978) and Von Braun (1991) use sphericalharmonic translation and rotation theorems to obtain an open-ended expansion relative to an inertial reference frame. Maciejewski (1995) simplifies the expansion by adopting a reference frame fixed in one body. Hartmann et al. (1994) formulate spherical harmonics in terms of symmetric, trace-free (STF) tensors. This formulation expresses in a very compact way the potential and force expressions, as well as mutual potential.

As another approach for mutual potential, Paul (1988) expands $1 / r$ in a six-dimensional Taylor series. Components of attitude matrices relating body-fixed and inertial coordinate systems appear explicitly. 'Inertia integrals' resembling $\iiint_{M} x^{i} y^{j} z^{k} \mathrm{~d} m$ are evaluated once in body-fixed coordinates, and can accommodate variable-density bodies. However, the formulation concludes with a total of 15 nested summations, the first six of which are infinite. 
In this paper we present a new series expansion for one special case, the mutual potential of two homogeneous polyhedra. Eliminating density variations simplifies the mathematics, at the expense of weakening the applicability to idealized bodies. However, choosing polyhedra as subject bodies increases the flexibility of the formalism over more specialized bodies such as triaxial ellipsoids. Faces can be large and small, and can directly represent important surface features such as deep clefts where contact binaries meet.

In the 19th century, Liebenthal (1880) likewise addressed the mutual potential of homogeneous polyhedra. This formalism, although incomplete, is in closed form. We summarize Liebenthal's pioneering work before presenting our own.

Iterated volume integrals are converted to surface integrals using the Gauss Divergence Theorem and a lesser-known companion, perhaps named the Gradient Theorem (Riley et al., 2002, Section 11.9.1):

$$
\begin{aligned}
U & =\iiint_{A} \iiint_{B} \frac{1}{r} \mathrm{~d} B \mathrm{~d} A \\
& =\iiint \iiint_{A} \nabla_{B} \cdot \frac{\mathbf{r}}{2 r} \mathrm{~d} B \mathrm{~d} A \\
& =\frac{1}{2} \iiint_{A} \iint_{\partial B} \hat{\mathbf{n}}_{\partial B} \cdot \frac{\mathbf{r}}{r} \mathrm{~d} \partial B \mathrm{~d} A \\
& =\frac{1}{2} \iint_{\partial B} \hat{\mathbf{n}}_{\partial B} \cdot \iiint_{A} \frac{\mathbf{r}}{r} \mathrm{~d} A \mathrm{~d} \partial B \\
& =\frac{1}{2} \iint_{\partial B} \hat{\mathbf{n}}_{\partial B} \cdot \iiint_{A} \nabla_{A}(-r) \mathrm{d} A \mathrm{~d} \partial B \\
& =-\frac{1}{2} \iint_{\partial B} \hat{\mathbf{n}}_{\partial B} \cdot \iint_{\partial A} \hat{\mathbf{n}}_{\partial A} r \mathrm{~d} \partial A \mathrm{~d} \partial B \\
& =-\frac{1}{2} \iint_{\partial B} \iint_{\partial A} \hat{\mathbf{n}}_{\partial A} \cdot \hat{\mathbf{n}}_{\partial B} r \mathrm{~d} \partial A \mathrm{~d} \partial B .
\end{aligned}
$$

Here, $\partial A$ and $\partial B$ represent the surfaces of bodies $A$ and $B, \hat{\mathbf{n}}$ is a surface normal vector, and $r$ is the distance between differential surface elements $\mathrm{d} \partial A$ and $\mathrm{d} \partial B$. The minus sign appears because both ends of relative position vector $\mathbf{r} \equiv \mathbf{r}_{B}-\mathbf{r}_{A}$ have been differentiated by the $\nabla_{A}$ and $\nabla_{B}$ operators.

Next, the bodies are specialized to polyhedra. The whole-surface integrations are broken into summations of integrations over face pairs $a$ and $b$, where surface normal vectors are constant: 


$$
U=-\frac{1}{2} \sum_{a \in \partial A} \sum_{b \in \partial B} \hat{\mathbf{n}}_{a} \cdot \hat{\mathbf{n}}_{b} \iint_{a} \iint_{b} r \mathrm{~d} b \mathrm{~d} a .
$$

One of the surface integrations is converted into a line integration around the boundary of the face. Liebenthal writes $r^{2}=\rho^{2}+p^{2}$ where $\rho$ is the distance in the face plane and $p$ is the out-of-plane coordinate. Then the Divergence Theorem is invoked using 2-D polar coordinates, where $\rho$ is the radial component of the vector field and there is no angular component:

$$
\begin{aligned}
\iint_{\text {face }} r \mathrm{~d} \sigma & =\iint_{\text {face }} \nabla_{2} \cdot\left[\left(\frac{1}{\rho} \int \rho r \mathrm{~d} \rho\right) \hat{\mathbf{e}}_{\rho}\right] \mathrm{d} \sigma \\
& =\oint_{\partial}\left(\frac{1}{\rho} \int \rho \sqrt{\rho^{2}+p^{2}} \mathrm{~d} \rho\right) \hat{\mathbf{n}}_{e} \cdot \hat{\mathbf{e}}_{\rho} \mathrm{d} s \\
& =\frac{1}{3} \oint_{\partial} \frac{\sqrt{\left(\rho^{2}+p^{2}\right)^{3}}}{\rho} \hat{\mathbf{n}}_{e} \cdot \hat{\mathbf{e}}_{\rho} \mathrm{d} s
\end{aligned}
$$

Next, the boundary integral is segmented into straight polygon edges indexed by $k$. In-plane distance $\rho$ is expressed in terms of coordinates $s_{k}$ along the edge and constant $q_{k}$ normal to it:

$$
\begin{aligned}
\iint_{\text {face }} r \mathrm{~d} \sigma= & \frac{1}{3} \sum_{k \in \text { edges }} \int_{k} \frac{\sqrt{\left(s_{k}^{2}+q_{k}^{2}+p^{2}\right)^{3}}}{\sqrt{s_{k}^{2}+q_{k}^{2}}} \frac{q_{k}}{\sqrt{s_{k}^{2}+q_{k}^{2}}} \mathrm{~d} s_{k} \\
= & \frac{1}{3} \sum_{k \in \text { edges }} q_{k} \int_{s_{k}^{\prime}}^{s_{k}^{\prime \prime}\left(s_{k}^{2}+q_{k}^{2}+p^{2}\right) \sqrt{s_{k}^{2}+q_{k}^{2}+p^{2}}} \frac{s_{k}^{2}+q_{k}^{2}}{\mathrm{~d}} s_{k} \\
= & \sum_{k \in \text { edges }}\left(q_{k} \frac{3 p^{2}+q_{k}^{2}}{6} \log \left(s_{k}+r_{k}\right)+\frac{q_{k} s_{k}}{6} r_{k}\right. \\
& \left.+\frac{p^{3}}{3} \arctan \frac{p s_{k}}{q_{k} r_{k}}\right)\left.\right|_{s_{k}^{\prime}, r_{k}^{\prime}} ^{s_{k}^{\prime \prime}, r_{k}^{\prime \prime}}
\end{aligned}
$$

Thus Liebenthal has accomplished one of the surface integrations analytically.

The remaining surface integration is intractable. There are radicals within the elementary functions, and a messy change of coordinates is involved as focus shifts to the remaining face. This is unfortunate; Equation (1) is appealing in its simplicity. What would Euler do?

One way to proceed is by numerical integration of the remaining surface integration. There are 'cubature' algorithms designed specifically for triangular integration domains (Berntsen and Espelid, 1992). 
In this paper, we evaluate mutual potential between homogeneous polyhedra in the following way. Each polyhedron is segmented into a collection of simplices (tetrahedra). The integrand $1 / r$ is expanded in a single infinite series of Legendre polynomials. Certain elementary values are expressed profitably in terms of 6-dimensional tensors. Both volume integrations over simplex pairs are accomplished directly using a result from Dirichlet. The result is a straightforward infinite series for the mutual potential.

\section{Integration over Two Polyhedra}

The problem is to evaluate a pair of iterated integrals over the volumes of bodies $A$ and $B$ :

$$
U \equiv \iiint_{A} \iiint_{B} \frac{1}{r} \mathrm{~d} B \mathrm{~d} A,
$$

where $r$ is the distance between differential volume elements $\mathrm{d} A$ and $\mathrm{d} B$. (We have omitted cluttering factors such as the gravitational constant and the two bodies' individual densities.)

When the bodies are polyhedra, we begin by segmenting each into a collection of simplices (tetrahedra). One vertex of each simplex is placed at the polyhedron's centroid. The other three simplex vertices are taken from the face vertices. With no loss of generality, we assume faces are triangular. Equation (2) becomes a summation of integrations over simplices $a$ and $b$ from the two bodies.

$$
U=\sum_{a \in A} \sum_{b \in B} \iiint_{a} \iiint_{b} \frac{1}{r} \mathrm{~d} b \mathrm{~d} a
$$

Absolute position vectors are reckoned from an inertially fixed origin (Figure 1). Let vector $\mathbf{A}=\left(x_{A}, y_{A}, z_{A}\right)$ be the centroid of body $A$, and let differential volume $\mathrm{d} A$ be located at absolute position $\mathbf{a}=\left(x_{a}, y_{a}, z_{a}\right)$ and at position $\mathbf{a}-\mathbf{A}=\left(\Delta x_{a}, \Delta y_{a}, \Delta z_{a}\right)$ relative to the centroid. Similar definitions hold for body $B$. (These $\Delta$ coordinates are expressed in the inertial coordinate system common to both bodies, rather than in body-fixed coordinate systems.)

The distance $r$ between differential volumes is

$$
\begin{aligned}
r^{2}= & \left(x_{a}-x_{b}\right)^{2}+\left(y_{a}-y_{b}\right)^{2}+\left(z_{a}-z_{b}\right)^{2} \\
= & {\left[\left(x_{A}-x_{B}\right)+\left(\Delta x_{a}-\Delta x_{b}\right)\right]^{2}+\left[\left(y_{A}-y_{B}\right)+\left(\Delta y_{a}-\Delta y_{b}\right)\right]^{2} } \\
& +\left[\left(z_{A}-z_{B}\right)+\left(\Delta z_{a}-\Delta z_{b}\right)\right]^{2} .
\end{aligned}
$$




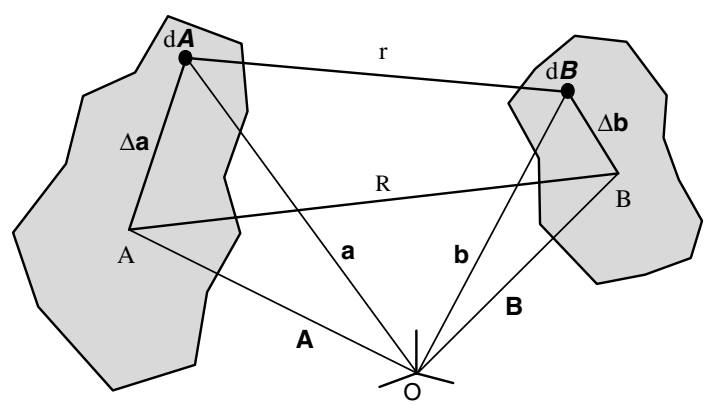

Figure 1. Vectors $\mathbf{A}$ and $\mathbf{B}$ locate the centroids of two bodies separated by distance $R$. Vectors $\mathbf{a}$ and $\mathbf{b}$ locate two differential volumes $\mathrm{d} A$ and $\mathrm{d} B$ separated by distance $r$.

To simplify the notation, we define vectors

$$
\begin{aligned}
\mathbf{R} & \equiv\left(x_{A}-x_{B}, y_{A}-y_{B}, z_{A}-z_{B}\right), \\
\mathbf{h} & \equiv\left(\Delta x_{a}-\Delta x_{b}, \Delta y_{a}-\Delta y_{b}, \Delta z_{a}-\Delta z_{b}\right),
\end{aligned}
$$

and their norms

$$
R^{2}=\mathbf{R} \cdot \mathbf{R}, \quad h^{2}=\mathbf{h} \cdot \mathbf{h} .
$$

Then $r$ can be written as

$$
\begin{aligned}
r^{2} & =(\mathbf{R}+\mathbf{h}) \cdot(\mathbf{R}+\mathbf{h}) \\
& =R^{2}+h^{2}+2 \mathbf{R} \cdot \mathbf{h} .
\end{aligned}
$$

Vector $\mathbf{R}$, of course, is the relative vector between the two centroids. Vector $\mathbf{h}$ is somewhat unusual because it incorporates two independent excursions, one on each end of vector $\mathbf{R}$ (Figure 2).

\section{Series Expansion}

We now use the traditional procedure to expand $1 / r$ as a series involving Legendre polynomials $P_{n}()$.

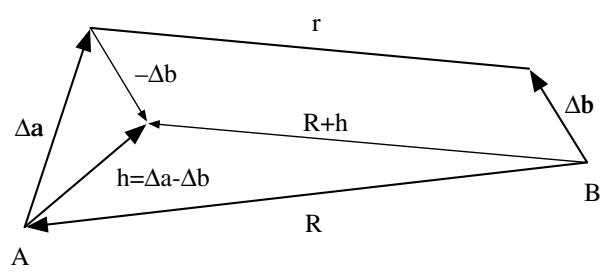

Figure 2. Vector $\mathbf{R}$ separates the bodies' centroids. Vector $\mathbf{h}$ is the difference of the differential volumes' offsets from their respective centroids. The magnitude of their sum $\mathbf{R}+\mathbf{h}$ is equivalent to $r$, the distance separating the differential volumes. 


$$
\begin{aligned}
1 / r= & \left(R^{2}+h^{2}+2 \mathbf{R} \cdot \mathbf{h}\right)^{-1 / 2} \\
= & \frac{1}{R}\left[1+\left(\frac{h}{R}\right)^{2}-2\left(\frac{h}{R}\right)\left(-\frac{\mathbf{R} \cdot \mathbf{h}}{R h}\right)\right]^{-1 / 2} \\
= & \frac{1}{R} \sum_{n=0}^{\infty}\left(\frac{h}{R}\right)^{n} P_{n}\left(-\frac{\mathbf{R} \cdot \mathbf{h}}{R h}\right) \\
= & {\left[\frac{1}{R}\right]+\left[-\frac{\mathbf{R} \cdot \mathbf{h}}{R^{3}}\right]+\left[-\frac{h^{2}}{2 R^{3}}+\frac{3(\mathbf{R} \cdot \mathbf{h})^{2}}{2 R^{5}}\right] } \\
& +\left[\frac{3 h^{2} \mathbf{R} \cdot \mathbf{h}}{2 R^{5}}-\frac{5(\mathbf{R} \cdot \mathbf{h})^{3}}{2 R^{7}}\right]+\cdots
\end{aligned}
$$

Individual terms in the series have been bracketed. Since $R$ appears in denominators, the two centroids cannot coincide, making $R=0$.

Convergence properties are dictated by $h / R$ in Equation (4). The maximum possible value of $h$ is the sum of the radii of the two bodies' Brillouin spheres. Hence, if the Brillouin spheres are disjoint, $h / R<1$ and the series converges.

When integrating Equation (5) in Equation (2), vector $\mathbf{R}$ and its norm $R$ remain constant. Only vector $\mathbf{h}$ and its norm $h$ vary. By inspection, Equation (5) contains no radicals involving these two. Hence, the Legendre expansion leads to tractable, radical-free integrands.

\section{Change of Variables}

To prepare for integration, we change variables to a barycenter formulation using $\left(u_{a}, v_{a}, w_{a}\right)$ for simplex $a$ and similarly for $b$. Range limits are $0 \leqslant\left(u_{a}, v_{a}, w_{a}, u_{a}+v_{a}+w_{a}\right) \leqslant 1$.

Given vertex coordinates

$$
\begin{aligned}
& \left(x_{1}^{a}, y_{1}^{a}, z_{1}^{a}\right)=\left(x_{A}, y_{A}, z_{A}\right)+\left(\Delta x_{1}^{a}, \Delta y_{1}^{a}, \Delta z_{1}^{a}\right), \\
& \left(x_{2}^{a}, y_{2}^{a}, z_{2}^{a}\right)=\left(x_{A}, y_{A}, z_{A}\right)+\left(\Delta x_{2}^{a}, \Delta y_{2}^{a}, \Delta z_{2}^{a}\right), \\
& \left(x_{3}^{a}, y_{3}^{a}, z_{3}^{a}\right)=\left(x_{A}, y_{A}, z_{A}\right)+\left(\Delta x_{3}^{a}, \Delta y_{3}^{a}, \Delta z_{3}^{a}\right),
\end{aligned}
$$

the coordinates of any point in simplex $a$ are

$$
\begin{aligned}
{\left[\begin{array}{l}
x_{a} \\
y_{a} \\
z_{a}
\end{array}\right] } & =\left[\begin{array}{l}
x_{A} \\
y_{A} \\
z_{A}
\end{array}\right]+u_{a}\left[\begin{array}{l}
\Delta x_{1}^{a} \\
\Delta y_{1}^{a} \\
\Delta z_{1}^{a}
\end{array}\right]+v_{a}\left[\begin{array}{l}
\Delta x_{2}^{a} \\
\Delta y_{2}^{a} \\
\Delta z_{2}^{a}
\end{array}\right]+w_{a}\left[\begin{array}{l}
\Delta x_{3}^{a} \\
\Delta y_{3}^{a} \\
\Delta z_{3}^{a}
\end{array}\right] \\
& =\left[\begin{array}{l}
x_{A} \\
y_{A} \\
z_{A}
\end{array}\right]+\left[\begin{array}{lll}
\Delta x_{1}^{a} & \Delta x_{2}^{a} & \Delta x_{3}^{a} \\
\Delta y_{1}^{a} & \Delta y_{2}^{a} & \Delta y_{3}^{a} \\
\Delta z_{1}^{a} & \Delta z_{2}^{a} & \Delta z_{3}^{a}
\end{array}\right]\left[\begin{array}{c}
u_{a} \\
v_{a} \\
w_{a}
\end{array}\right] .
\end{aligned}
$$


Changing integration variables requires introducing the Jacobian determinant of the transformation. It is simply

$$
T_{a} \equiv \frac{\partial\left(x_{a}, y_{a}, z_{a}\right)}{\partial\left(u_{a}, v_{a}, w_{a}\right)}=\operatorname{det}\left[\begin{array}{ccc}
\Delta x_{1}^{a} & \Delta x_{2}^{a} & \Delta x_{3}^{a} \\
\Delta y_{1}^{a} & \Delta y_{2}^{a} & \Delta y_{3}^{a} \\
\Delta z_{1}^{a} & \Delta z_{2}^{a} & \Delta z_{3}^{a}
\end{array}\right],
$$

with a similar expression for $T_{b}$. This is independent of the integration variables $u_{a}, \ldots, w_{b}$ and can be moved outside of the integrations.

The change to barycenter variables transforms each simplex $a$ and $b$ into a 'standard' simplex $a^{\prime}$ and $b^{\prime}$ with vertex coordinates $(0,0,0),(1,0,0),(0,1,0)$, and $(0,0,1)$.

$$
U=\sum_{a \in A} \sum_{b \in B} T_{a} T_{b} \iiint_{a^{\prime}} \iiint_{b^{\prime}} \frac{1}{r} \mathrm{~d} b^{\prime} \mathrm{d} a^{\prime}
$$

Either volume integration now resembles

$$
\int_{0}^{1} \int_{0}^{1-u} \int_{0}^{1-u-v} \cdots \mathrm{d} w \mathrm{~d} v \mathrm{~d} u
$$

which we subsequently abbreviate to

$$
\iiint_{01} \ldots \mathrm{d} w \mathrm{~d} v \mathrm{~d} u
$$

\section{Intermediate Expressions}

Due to the infinite sequence of degrees appearing in Equation (5), vector or matrix notations are inadequate. We adopt tensor notation and the Einstein summation convention. No significance should be attributed to the distinction of superscript and subscript indices; we are not claiming covariant and contravariant behavior. We use tensor notation in a very elementary way, as a bookkeeping device for inner and outer products. Our space is sixdimensional, three each from simplices $a$ and $b$.

First we define three 6-vectors containing vertex coordinates relative to each body's centroid. In them, we give negative weight to simplex $b$ :

$$
\begin{aligned}
\mathbf{x}^{i} & \equiv\left[\Delta x_{1}^{a}, \Delta x_{2}^{a}, \Delta x_{3}^{a},-\Delta x_{1}^{b},-\Delta x_{2}^{b},-\Delta x_{3}^{b}\right], \\
\mathbf{y}^{i} & \equiv\left[\Delta y_{1}^{a}, \Delta y_{2}^{a}, \Delta y_{3}^{a},-\Delta y_{1}^{b},-\Delta y_{2}^{b},-\Delta y_{3}^{b}\right], \\
\mathbf{z}^{i} & \equiv\left[\Delta z_{1}^{a}, \Delta z_{2}^{a}, \Delta z_{3}^{a},-\Delta z_{1}^{b},-\Delta z_{2}^{b},-\Delta z_{3}^{b}\right] .
\end{aligned}
$$


And then we stack all into a $3 \times 6$ matrix.

$$
\mathbf{v}_{j}^{i} \equiv\left[\begin{array}{c}
\Delta x_{1}^{a}, \Delta x_{2}^{a}, \Delta x_{3}^{a},-\Delta x_{1}^{b},-\Delta x_{2}^{b},-\Delta x_{3}^{b} \\
\Delta y_{1}^{a}, \Delta y_{2}^{a}, \Delta y_{3}^{a},-\Delta y_{1}^{b},-\Delta y_{2}^{b},-\Delta y_{3}^{b} \\
\Delta z_{1}^{a}, \Delta z_{2}^{a}, \Delta z_{3}^{a},-\Delta z_{1}^{b},-\Delta z_{2}^{b},-\Delta z_{3}^{b}
\end{array}\right]=\left[\begin{array}{c}
\mathbf{x}_{i} \\
\mathbf{y}_{i} \\
\mathbf{z}_{i}
\end{array}\right]_{j}
$$

The superscript index varies $1 \ldots 6$ and the subscript $1 \ldots 3$.

We also define a kernel 6-vector

$$
\mathbf{q}_{i} \equiv\left[u_{a}, v_{a}, w_{a}, u_{b}, v_{b}, w_{b}\right]
$$

containing the barycenter variables.

Each term in Equation (5) consists of factors $(\mathbf{R} \cdot \mathbf{h}),\left(h^{2}\right)$, and others that are constant during integration. The two that vary can be expressed using the kernel. First, the components of 3 -vector $\mathbf{h}$ are

$$
\begin{aligned}
\mathbf{h}_{j} & =\left[\mathbf{q}_{i} \mathbf{x}^{i}, \mathbf{q}_{i} \mathbf{y}^{i}, \mathbf{q}_{i} \mathbf{z}^{i}\right]_{j} \\
& =\mathbf{q}_{i} \mathbf{v}_{j}^{i} .
\end{aligned}
$$

Next,

$$
\begin{aligned}
\mathbf{R} \cdot \mathbf{h} & =\mathbf{R}^{j} \mathbf{h}_{j} \\
& =\mathbf{R}^{j} \mathbf{q}_{i} \mathbf{v}_{j}^{i} \\
& =\left[\begin{array}{l}
x_{A}-x_{B} \\
y_{A}-y_{B} \\
z_{A}-z_{B}
\end{array}\right] \cdot\left[\begin{array}{l}
\mathbf{q}_{i} \mathbf{x}^{i} \\
\mathbf{q}_{i} \mathbf{y}^{i} \\
\mathbf{q}_{i} \mathbf{z}^{i}
\end{array}\right] \\
& =\mathbf{q}_{i}\left\{\left(x_{A}-x_{B}\right) \mathbf{x}^{i}+\left(y_{A}-y_{B}\right) \mathbf{y}^{i}+\left(z_{A}-z_{B}\right) \mathbf{z}^{i}\right\} \\
& \equiv \mathbf{q}_{i} \mathbf{w}^{i} .
\end{aligned}
$$

We have defined a 6-vector $\mathbf{w}^{i} \equiv \mathbf{R}^{j} \mathbf{v}_{j}^{i}$ for convenience.

Finally,

$$
\begin{aligned}
h^{2} & =\mathbf{h}_{k} \mathbf{h}_{k} \\
& =\mathbf{q}_{i} \mathbf{v}_{k}^{i} \mathbf{q}_{j} j_{k}^{j} \\
& =\mathbf{q}_{i} \mathbf{q}_{j} \mathbf{x}^{i} \mathbf{x}^{j}+\mathbf{q}_{i} \mathbf{q}_{j} \mathbf{y}^{i} \mathbf{y}^{j}+\mathbf{q}_{i} \mathbf{q}_{j} \mathbf{z}^{i} \mathbf{z}^{j} \\
& =\left(\mathbf{q}_{i} \mathbf{q}_{j}\right)\left(\mathbf{x}^{i} \mathbf{x}^{j}+\mathbf{y}^{i} \mathbf{y}^{j}+\mathbf{z}^{i} \mathbf{z}^{j}\right) \\
& \equiv \mathbf{q}_{i j} \mathbf{r}^{i j} .
\end{aligned}
$$

Here we define the rank-2 tensor $\mathbf{q}_{i j} \equiv \mathbf{q}_{i} \mathbf{q}_{j}$ as the outer product of the kernel with itself. We subsequently generalize this to rank- $k$ tensors:

$$
\mathbf{q}_{i_{1} i_{2} \ldots i_{k}} \equiv \mathbf{q}_{i_{1}} \mathbf{q}_{i_{2}} \ldots \mathbf{q}_{i_{k}} .
$$

We have also defined another rank-2 tensor (in this case, a $6 \times 6$ matrix):

$$
\begin{aligned}
\mathbf{r}^{i j} & \equiv \mathbf{x}^{i} \mathbf{x}^{j}+\mathbf{y}^{i} \mathbf{y}^{j}+\mathbf{z}^{i} \mathbf{z}^{j} \\
& =\mathbf{v}_{k}^{i} \mathbf{v}_{k}^{j} .
\end{aligned}
$$


Both $\mathbf{r}^{i j}$ and $\mathbf{w}^{i}$ vary with any orientation change of the bodies. However, they are constant during integration.

\section{Termwise Integration of the Legendre Expansion}

Terms in Equation (5) contain $(\mathbf{R} \cdot \mathbf{h})$ and $\left(h^{2}\right)$ factors. We substitute the corresponding expressions (Equations 10,11) and group all kernel vectors into one tensor $\mathbf{q}_{i_{1} \ldots i_{k}}$ of outer products. The integration variables $u_{a}, \ldots, w_{b}$ are completely isolated to it.

For example, $\left(h^{2}\right)(\mathbf{R} \cdot \mathbf{h})$ appears in the third-degree term of Equation (5). We determine the following.

$$
\begin{aligned}
\iiint_{a} \iiint_{b}\left(h^{2}\right)(\mathbf{R} \cdot \mathbf{h}) \mathrm{d} b \mathrm{~d} a & =T_{a} T_{b} \iiint_{01} \iiint_{01}\left(\mathbf{q}_{i j} \mathbf{r}^{i j}\right)\left(\mathbf{q}_{k} \mathbf{w}^{k}\right) \mathrm{d} b^{\prime} \mathrm{d} a^{\prime} \\
& =\left(\iiint \iiint_{01} \mathbf{q}_{i j k} \mathrm{~d} b^{\prime} \mathrm{d} a^{\prime}\right) \mathbf{r}^{i j} \mathbf{w}^{k} T_{a} T_{b} .
\end{aligned}
$$

Each component of the integrand $\mathbf{q}_{i_{1} \ldots i_{k}}$ resembles

$$
u_{a}^{I_{a}} v_{a}^{J_{a}} w_{a}^{K_{a}} u_{b}^{I_{b}} v_{b}^{J_{b}} w_{b}^{K_{b}}
$$

where exponents $I_{a}, \ldots, K_{b}$ are nonnegative integers. An insight leads to a straightforward way of determining exponents. In effect, we take the logarithm of each component by bookkeeping only the exponents. That is, the kernel vector

$$
\mathbf{q}_{i}=\left[\begin{array}{c}
u_{a} \\
v_{a} \\
w_{a} \\
u_{b} \\
v_{b} \\
w_{b}
\end{array}\right]=\left[\begin{array}{c}
u_{a}^{1} v_{a}^{0} w_{a}^{0} u_{b}^{0} v_{b}^{0} w_{b}^{0} \\
u_{a}^{0} v_{a}^{1} w_{a}^{0} u_{b}^{0} v_{b}^{0} w_{b}^{0} \\
u_{a}^{0} v_{a}^{0} w_{a}^{1} u_{b}^{0} v_{b}^{0} w_{b}^{0} \\
u_{a}^{0} v_{a}^{0} w_{a}^{0} u_{b}^{1} v_{b}^{0} w_{b}^{0} \\
u_{a}^{0} v_{a}^{0} w_{a}^{0} u_{b}^{0} v_{b}^{1} w_{b}^{0} \\
u_{a}^{0} v_{a}^{0} w_{a}^{0} u_{b}^{0} v_{b}^{0} w_{b}^{1}
\end{array}\right]
$$

is rewritten as

$$
\overline{\mathbf{q}}_{i}=\left[\begin{array}{cccccc}
(1 & 0 & 0 & 0 & 0 & 0) \\
(0 & 1 & 0 & 0 & 0 & 0) \\
(0 & 0 & 1 & 0 & 0 & 0) \\
(0 & 0 & 0 & 1 & 0 & 0) \\
(0 & 0 & 0 & 0 & 1 & 0) \\
(0 & 0 & 0 & 0 & 0 & 1)
\end{array}\right] .
$$


When forming a rank- $k$ integrand, what was taking the outer product of the kernels becomes taking the 'outer sum' of the exponents. For example, here are exponents of the rank-2 integrand $\mathbf{q}_{i j}=\mathbf{q}_{i} \mathbf{q}_{j}$ :

$$
\overline{\mathbf{q}}_{i j}=\left[\begin{array}{rrrrrr}
(200000) & (110000) & (101000) & (100100) & (100010) & (100001) \\
(110000) & (020000) & (011000) & (010100) & (010010) & (010001) \\
(101000) & (011000) & (002000) & (001100) & (001010) & (001001) \\
(100100) & (010100) & (001100) & (000200) & (000110) & (000101) \\
(100010) & (010010) & (001010) & (000110) & (000020) & (000011) \\
(100001) & (010001) & (001001) & (000101) & (000011) & (000002)
\end{array}\right] .
$$

Since variable sets $\left(u_{a}, v_{a}, w_{a}\right)$ and $\left(u_{b}, v_{b}, w_{b}\right)$ are independent, the iterated volume integrations separate into a pair of factors, each resembling

$$
\int_{0}^{1} \int_{0}^{1-u} \int_{0}^{1-u-v} u^{I} v^{J} w^{K} \mathrm{~d} w \mathrm{~d} v \mathrm{~d} u=\frac{I ! J ! K !}{(I+J+K+3) !}
$$

an instance of the Dirichlet integral (Macmillan, 1930, Section 50).

We denote the result with a capital letter:

$$
\mathbf{Q}_{i_{1} \ldots i_{k}} \equiv \iiint_{01} \iiint_{01} \mathbf{q}_{i_{1} \ldots i_{k}} \mathrm{~d} b^{\prime} \mathrm{d} a^{\prime}
$$

Each is a rank- $k$ tensor of rational numbers, symmetric in all index pairs. Here are the first four $\mathbf{Q}_{i_{1} \ldots i_{k}}$ tensors.

$$
\begin{aligned}
& \mathbf{Q}=\frac{1}{36}, \quad \mathbf{Q}_{i}=\frac{1}{144}\left[\begin{array}{llllll}
1 & 1 & 1 & 1 & 1 & 1
\end{array}\right], \\
& \mathbf{Q}_{i j}=\frac{1}{2880}\left[\begin{array}{llllll}
8 & 4 & 4 & 5 & 5 & 5 \\
4 & 8 & 4 & 5 & 5 & 5 \\
4 & 4 & 8 & 5 & 5 & 5 \\
5 & 5 & 5 & 8 & 4 & 4 \\
5 & 5 & 5 & 4 & 8 & 4 \\
5 & 5 & 5 & 4 & 4 & 8
\end{array}\right] \\
& \mathbf{Q}_{i j k}=\frac{1}{8640}\left(\left[\begin{array}{cccccc}
12 & 4 & 4 & 6 & 6 & 6 \\
4 & 4 & 2 & 3 & 3 & 3 \\
4 & 2 & 4 & 3 & 3 & 3 \\
6 & 3 & 3 & 6 & 3 & 3 \\
6 & 3 & 3 & 3 & 6 & 3 \\
6 & 3 & 3 & 3 & 3 & 6
\end{array}\right]\left[\begin{array}{cccccc}
4 & 4 & 2 & 3 & 3 & 3 \\
4 & 12 & 4 & 6 & 6 & 6 \\
2 & 4 & 4 & 3 & 3 & 3 \\
3 & 6 & 3 & 6 & 3 & 3 \\
3 & 6 & 3 & 3 & 6 & 3 \\
3 & 6 & 3 & 3 & 3 & 6
\end{array}\right]\left[\begin{array}{cccccc}
4 & 2 & 4 & 3 & 3 & 3 \\
2 & 4 & 4 & 3 & 3 & 3 \\
4 & 4 & 12 & 6 & 6 & 6 \\
3 & 3 & 6 & 6 & 3 & 3 \\
3 & 3 & 6 & 3 & 6 & 3 \\
3 & 3 & 6 & 3 & 3 & 6
\end{array}\right]\right. \\
& \left.\left[\begin{array}{llllll}
6 & 3 & 3 & 6 & 3 & 3 \\
3 & 6 & 3 & 6 & 3 & 3 \\
3 & 3 & 6 & 6 & 3 & 3 \\
6 & 6 & 6 & 12 & 4 & 4 \\
3 & 3 & 3 & 4 & 4 & 2 \\
3 & 3 & 3 & 4 & 2 & 4
\end{array}\right]\left[\begin{array}{llllll}
6 & 3 & 3 & 3 & 6 & 3 \\
3 & 6 & 3 & 3 & 6 & 3 \\
3 & 3 & 6 & 3 & 6 & 3 \\
3 & 3 & 3 & 4 & 4 & 2 \\
6 & 6 & 6 & 4 & 12 & 4 \\
3 & 3 & 3 & 2 & 4 & 4
\end{array}\right]\left[\begin{array}{llllll}
6 & 3 & 3 & 3 & 3 & 6 \\
3 & 6 & 3 & 3 & 3 & 6 \\
3 & 3 & 6 & 3 & 3 & 6 \\
3 & 3 & 3 & 4 & 2 & 4 \\
3 & 3 & 3 & 2 & 4 & 4 \\
6 & 6 & 6 & 4 & 4 & 12
\end{array}\right]\right)
\end{aligned}
$$




\section{Simplifications}

We gather intermediate results and display the iterated volume integrals:

$$
\begin{aligned}
\iiint_{01} \iiint_{01} \frac{1}{r} \mathrm{~d} b^{\prime} \mathrm{d} a^{\prime}= & {\left[\frac{\mathbf{Q}}{R}\right]+\left[-\frac{\mathbf{Q}_{i} \mathbf{w}^{i}}{R^{3}}\right]+\left[-\frac{\mathbf{Q}_{i j} \mathbf{r}^{i j}}{2 R^{3}}+\frac{3 \mathbf{Q}_{i j} \mathbf{w}^{i} \mathbf{w}^{j}}{2 R^{5}}\right] } \\
& +\left[\frac{3 \mathbf{Q}_{i j k} \mathbf{r}^{i j} \mathbf{w}^{k}}{2 R^{5}}-\frac{5 \mathbf{Q}_{i j k} \mathbf{w}^{i} \mathbf{w}^{j} \mathbf{w}^{k}}{2 R^{7}}\right]+\cdots
\end{aligned}
$$

This is ready to substitute into Equation (7), where it is understood that $\mathbf{r}^{i j}$ and $\mathbf{w}^{i}$ depend on the simplices $a$ and $b$.

When accumulated over all simplices, the leading term $\mathbf{Q} / R$ becomes the Keplerian term $U_{0}$ of the potential expansion:

$$
\begin{aligned}
U_{0} & =\sum_{a} \sum_{b} \frac{\mathbf{Q}}{R} T_{a} T_{b} \\
& =\frac{1}{R}\left(\sum_{a} \frac{1}{6} T_{a}\right)\left(\sum_{b} \frac{1}{6} T_{b}\right) \\
& =\frac{1}{R} V_{A} V_{B},
\end{aligned}
$$

where $V_{A}$ and $V_{B}$ are the volumes of the polyhedra.

The first-degree term $-\mathbf{Q}_{i} \mathbf{w}^{i} / R^{3}$ vanishes when the expansion centers $\mathbf{A}$ and $\mathbf{B}$ are the respective body centroids. To show this, we expand $\mathbf{Q}_{i} \mathbf{w}^{i}$ upon summation over the two bodies.

$$
\begin{aligned}
\sum_{a} \sum_{b} T_{a} T_{b} \mathbf{Q}_{i} \mathbf{w}^{i}= & \frac{1}{144} \sum_{a} \sum_{b} T_{a} T_{b} \\
& \times\left\{\begin{array}{c}
\left(x_{A}-x_{B}\right)\left(\Delta x_{1}^{a}+\Delta x_{2}^{a}+\Delta x_{3}^{a}-\Delta x_{1}^{b}-\Delta x_{2}^{b}-\Delta x_{3}^{b}\right) \\
+\left(y_{A}-y_{B}\right)\left(\Delta y_{1}^{a}+\Delta y_{2}^{a}+\Delta y_{3}^{a}-\Delta y_{1}^{b}-\Delta y_{2}^{b}-\Delta y_{3}^{b}\right) \\
+\left(z_{A}-z_{B}\right)\left(\Delta z_{1}^{a}+\Delta z_{2}^{a}+\Delta z_{3}^{a}-\Delta z_{1}^{b}-\Delta z_{2}^{b}-\Delta z_{3}^{b}\right)
\end{array}\right\} \\
= & \frac{1}{6}\left(x_{A}-x_{B}\right) \sum_{b} T_{b}\left[\sum_{a} \frac{1}{24}\left(\Delta x_{1}^{a}+\Delta x_{2}^{a}+\Delta x_{3}^{a}\right) T_{a}\right] \\
& -\frac{1}{6}\left(x_{A}-x_{B}\right) \sum_{a} T_{a}\left[\sum_{b} \frac{1}{24}\left(\Delta x_{1}^{b}+\Delta x_{2}^{b}+\Delta x_{3}^{b}\right) T_{b}\right] \\
& +\cdots .
\end{aligned}
$$

The bracketed factors are the bodies' first moments which vanish when the expansion centers are body centroids. 


\section{Conclusion}

The mutual potential $U$ between two homogeneous polyhedra $A$ and $B$ has been evaluated analytically. The resulting infinite series begins

$$
\begin{aligned}
U=\frac{V_{A} V_{B}}{R}+\sum_{a \in A} \sum_{a \in B} T_{a} T_{b}\{ & {\left[-\frac{\mathbf{Q}_{i j} \mathbf{r}^{i j}}{2 R^{3}}+\frac{3 \mathbf{Q}_{i j} \mathbf{w}^{i} \mathbf{w}^{j}}{2 R^{5}}\right] } \\
& \left.+\left[\frac{3 \mathbf{Q}_{i j k} \mathbf{r}^{i j} \mathbf{w}^{k}}{2 R^{5}}-\frac{5 \mathbf{Q}_{i j k} \mathbf{w}^{i} \mathbf{w}^{j} \mathbf{w}^{k}}{2 R^{7}}\right]+\cdots\right\} .
\end{aligned}
$$

(The gravitational constant $G$ and the two bodies' individual densities have been omitted.) $R$ is the distance separating the centroids, and $V_{A}$ and $V_{B}$ represent the volumes. The degree- 1 term has vanished because polyhedra centroids were used as expansion centers for the series.

Within the summations, $a$ and $b$ represent simplices comprising the two polyhedra, and $T_{a}$ and $T_{b}$ are Jacobian determinants given in Equation (6).

The $\mathbf{Q}$ are multi-rank, symmetric tensors containing constant rational expressions. See Equation (13) and vicinity. They represent generic results of the iterated volume integrations.

Intermediate vector $\mathbf{w}^{i}=\mathbf{R}^{j} \mathbf{v}_{j}^{i}$ and matrix $\mathbf{r}^{i j}=\mathbf{v}_{k}^{i} \mathbf{v}_{k}^{j}$ (Equations 9, 12) both depend on simplices $a$ and $b$, even though the notation does not show this. Vector $\mathbf{R}^{j}$ in Equation (3) contains the centroid separation coordinates, and matrix $\mathbf{v}_{j}^{i}$ in Equation (8) contains vertex coordinates relative to centroids, with body $B$ having negative weight.

Note the absence of special functions in our final Equation (14). By contrast, spherical-harmonic formulations involve Legendre polynomials and associated Legendre functions, while Liebenthal's result contains $\log$ and arctan functions. This simplicity can speed computer evaluation.

There are drawbacks to our formulation. At the onset we assume homogenous bodies without density variations. The result is not in closed form, being derived from an infinite series expansion. There is no mention of attitude matrices relating body-fixed and inertial coordinates; summations over the simplices must be reevaluated with any orientation change. Without taking advantage of symmetries and constant subexpressions, the work to evaluate the $n$th degree term in Equation (14) is $O\left(6^{n}\right)$, increasing steeply with $n$.

The procedures for computing force and torque in Maciejewski (1995) are appropriate for our formulation. Forces are computed by differentiating the mutual potential with respect to the centroid coordinates of each body. In Equation (14), this means differentiating $R$ and $\mathbf{w}^{i}$. Torques involve differentiating $\mathbf{x}^{i}, \mathbf{y}^{i}$, and $\mathbf{z}^{i}$, i.e. both $\mathbf{r}^{i j}$ and $\mathbf{w}^{i}$. 


\section{Acknowledgements}

The research described in this paper was carried out at the Jet Propulsion laboratory, California Institute of Technology, under a contract with the National Aeronautics and Space Administration.

\section{References}

Berntsen, J. and Espelid, T. O.: 1992, 'Algorithm 706 DCUTRI: An algorithm for adaptive volume cubature over a collection of triangles', ACM Trans. Math. Software 18(3), 329-342.

Borderies, N.: 1978, 'Mutual gravitational potential of N solid bodies', Celest. Mech. 18(3), 295-307.

Hartmann, T., Stoffel, M. and Kioustelidis, T.: 1994, 'On the use of STF-tensors in celestial mechanics', Celest. Mech. Dynam. Astron. 60, 139-159.

Liebenthal, E.: 1880, 'Untersuchungen über die Attraction zweier homogener Körper', PhD thesis, Universität Greifswald.

Maciejewski, A. J.: 1995, 'Reduction, relative equilibria and potential in the two rigid bodies problem', Celest. Mech. Dynam. Astron. 63(1), 1-28.

Macmillan, W. D.: 1930, The Theory of the Potential. McGraw Hill. Republished by Dover, New York, 1958.

Paul, M. K.: 1988, 'An expansion in power series of mutual potential for gravitating bodies with finite sizes', Celest. Mech. 44, 49-59.

Riley, K. F., Hobson, M. P. and Bence, S. J.: 2002, Mathematical Methods for Physics and Engineering, 2nd edn, Cambridge, Cambridge University Press, UK.

Von Braun, C.: 1991, 'The gravitational potential of two arbitrary, rotating bodies with applications to the Earth-Moon system', PhD thesis, The University of Texas at Austin, Austin, Texas. 